\title{
Sharply concentrated cosmic-ray excess fluxes from heliomagnetospheric nose and tail boundaries observed with neutron monitors on the ground
}

\author{
K. Nagashima ${ }^{1}$, I. Kondo ${ }^{2}$, and Z. Fujii ${ }^{1}$ \\ ${ }^{1}$ Solar-Terrestrial Environment Laboratory, Nagoya University, Chikusa-ku, Nagoya 464-8601, Japan \\ ${ }^{2}$ Institute for Cosmic Ray Research,University of Tokyo, 5-1-5 Kashinoha, Kashiwa 277-8582, Japan \\ (Received January 19, 2005; Revised July 21, 2005; Accepted August 2, 2005)
}

\begin{abstract}
Two kinds of sharply concentrated excess flux of cosmic rays from heliomagnetospheric nose and tail directions (right ascension $\alpha \sim 18$ hours and $\sim 6$ hours) are found by the analysis of sidereal daily variation of neutron intensity (median energy $E_{m} \sim 20 \mathrm{GeV}$ ) on the ground. These fluxes do not show any response to the polarity reversal of solar magnetic field at the north pole and is contradictory to the simulation of the solar modulation of galactic anisotropy, which produces sidereal variation at the Earth greater in the negative polarity state than in the positive state. This indicates that they are not of the galactic origin and would be produced on the heliomagnetospheric nose and tail boundaries where it is considered that the interaction between the galactic and solar magnetic fields could produce the cosmic-ray acceleration. The acceleration mechanism producing the polarity-independent sidereal variation against solar modulation will be discussed.
\end{abstract}

Key words: Cosmic-ray sidereal anisotropy, heliomagnetosphere (HMS), polarity of HMS, solar modulation.

\section{Introduction}

The existence of two kinds of cosmic-ray anisotropy (or excess flux) in the energy region less than $10^{4} \mathrm{GeV}$ was reported in previous papers (Nagashima et al., 1995, 1998). One of them is the galactic anisotropy with the deficit intensity in the direction with right ascension $\alpha_{G}=12$ hours and declination $\delta_{G}=20^{\circ}$ and the other is the heliotail-in anisotropy with excess intensity in the direction of $\alpha_{T} \sim 6$ hours and $\delta_{T} \sim-24^{\circ}$. The discovery of these anisotropies was enabled by the use of the hourly display of the cosmicray sidereal daily variations instead of their first and/or second harmonic components reported in the past (e.g. Nagashima and Mori, 1976; Kiraly et al., 1979; Jacklyn, 1986; Mori et al., 1989, and references therein). One of the key variations for the discovery was the one with the maximum phase at $\sim 6$ hours having been observed since 1961 with the underground muon telescope $\left(E_{m} \sim 184 \mathrm{GeV}\right)$ at Hobart (geographic latitude $\lambda=43^{\circ}$, longitude $\varphi=147^{\circ} \mathrm{E}$ ), (Fenton et al., 1961; Jacklyn, 1966, 1986).

The observation showed that the sidereal variation $G(t)$ produced by the galactic anisotropy coincides in shape with that observed with the air showers at Mt. Norikura $\left(E_{m} \sim\right.$ $1.5 \cdot 10^{4} \mathrm{GeV}$; Nagashima et al., 1998, hereafter called Ref. 1). Consequently, the solar modulation of $G(t)$ in low energy region $(E<500 \mathrm{GeV}$ ) was supposed to be not so severe as that estimated from the simulation (Nagashima $e t$ al., 1981, 1982), being in good agreement with the muon observation at Ottawa $\left(E_{m} \sim 90 \sim 660 \mathrm{GeV} ; \lambda=45^{\circ}\right.$, $\left.\varphi=76^{\circ} \mathrm{W}\right)$ in the period of 1976-1984, which showed no response of $G(t)$ to the polarity reversal of the solar

Copyright (c) The Society of Geomagnetism and Earth, Planetary and Space Sciences (SGEPSS); The Seismological Society of Japan; The Volcanological Society of Japan; The Geodetic Society of Japan; The Japanese Society for Planetary Sciences; TERRAPUB magnetic field at the north pole (Bercovitch, 1984).

However, after the lapse of about two decades since the Ottawa observations, the polarity dependence of $G(t)$ has been found in 2004 by the muon observations at Sakashita underground station $\left(\lambda=36^{\circ} \mathrm{N}, \varphi=138^{\circ} \mathrm{E}, E_{m} \sim 340 \sim\right.$ $650 \mathrm{GeV})$ in the period 1971-2000 owing to the accumulation of data. The variation $G(t)$ produced by the galactic anisotropy is greater in the negative polarity state than in the positive state as expected from the simulation of the solar modulation, but the one produced by the tail-in anisotropy does not show such a polarity dependence (Nagashima $e t$ $a l ., 2004)$. This indicates that $G(t)$ is exactly of galactic origin, but the tail-in anisotropy is not and would be produced on the boundary of the heliomagnetosphere (called HMS hereafter) where the interaction between the galactic and solar magnetic fields is thought to produce the cosmicray acceleration.

In order to confirm the existence of these anisotropies and their solar modulation in lower energy region $\left(E_{m} \sim 20\right.$ $\mathrm{GeV}$ ), the previous analysis of the sidereal variation of neutron component (Nagashima et al., 1983) has been reexamined. As the result, it is found that the variation contains two components produced respectively by two kinds of sharply concentrated excess flux from the tail and nose directions ( $\alpha \sim 6$ hours and $\sim 18$ hours) of HMS. The former flux is that found in the previous analysis and corresponds to the tail-in anisotropy having been observed in high energy region $(\sim 400 \mathrm{GeV})$ and the latter is a new anisotropy found in the present analysis. Although these fluxes arrive at the Earth respectively from opposite directions, they can be discriminated by observation owing to their sharp concentration. Similar to the tail-in anisotropy in the high energy region, these fluxes do not show any polarity dependence due to solar modulation, suggesting 
their helioboundary origin. The mechanism producing such a polarity-independence against solar modulation is discussed on the basis of the simulation of solar modulation of the anisotropy (cf. Nagashima et al., 1981, 1982; Nagashima and Morishita, 1983; Yasue et al., 1983).

\section{Analysis}

Neutron monitor data of 1,395 station-years at 74 stations in the period 1960-2000, compiled by WDC-C2 Center (Solar-Terrestrial Environment Laboratory, Nagoya University/Department of Environmental Sciences, Ibaraki University) are used in the present analysis. The sidereal daily variation $i(t)$ at each station is derived every calendar year from those data which do not contain incomplete solar daily variations nor abnormally large fluctuations such as GLE (Ground Level Enhancement of cosmic rays) and so forth. $i(t)$ is influenced by the annual variation (1 cycle/year) of the solar daily variation (365 cycles/year) connected with the orbital motion of the Earth around the sun. The influence is eliminated by the method using the anti-sidereal daily variation (364 cycles/year), (cf. Ref. 1). The average of $i(t) \mathrm{s}$ in some period at a station or all the stations is expressed as $I(t)$.

In order to study the origin of cosmic-ray anisotropy responsible for the sidereal variation by means of its solar modulation, $I(t)$ is classified into $I(t, Y) \mathrm{s}$ in four periods $(Y=A P, Q P, A N$ and $Q N)$. The symbols $A$ and $Q$ express respectively the active and quiet periods in solar cycle divided as equally as possible in considering solar actively. $P$ and $Q$ are the periods of positive and negative polarity state of the solar magnetic field at the north pole quoted from Howard (1974) and Solar Geophysical Data, U.S. Dep. of Commerce. The combined periods in the above express respectively 'Active and Positive' period, 'Quiet and Positive' period and so forth. The variation averaged over all the period is expressed as $I(t, A L L)$.

$I(t, A L L)$ and $I(t, Y)$ do not exactly reflect the source flux, because they are respectively the averages of $i(t) \mathrm{s}$ which have different maximum phases caused by the difference of the cosmic-ray geomagnetic deflection at different stations. However, as the averaged geographic latitude $\left|\lambda_{\text {stn }}\right|$ of the locations at all the stations with the weight of their observation periods is $\sim 49^{\circ}$, those variations can be regarded approximately as the variation observed at $\left|\lambda_{\text {stn }}\right|$.

\section{Sidereal Daily Variation}

Figures 1(a) and 1(b) show respectively the averaged sidereal daily variation $I(t, A L L)$ and the histogram of the maximum phase $\left(t_{\max }\right)$ of the first harmonic component of $i(t)$ for all the stations in all the period. $I(t, A L L)$ shows a remarkable peak at $\sim 6$ hours in local sidereal time. As mentioned in Introduction, this peak was found long ago with underground muon telescope at Hobart (Jacklyn, 1966) and by the harmonic analysis of neutron monitor data of 620 station-years during the period 1958-1979 (Nagashima et al., 1983), and has been recently interpreted as being due to the tail-in anisotropy (Ref. 1). Hereafter, the sidereal daily variation produced by the tail-in anisotropy is expressed as $T(t)$. In addition to $T(t), I(t)$ contains also another variation which is almost submerged beneath $T(t)$ and can be

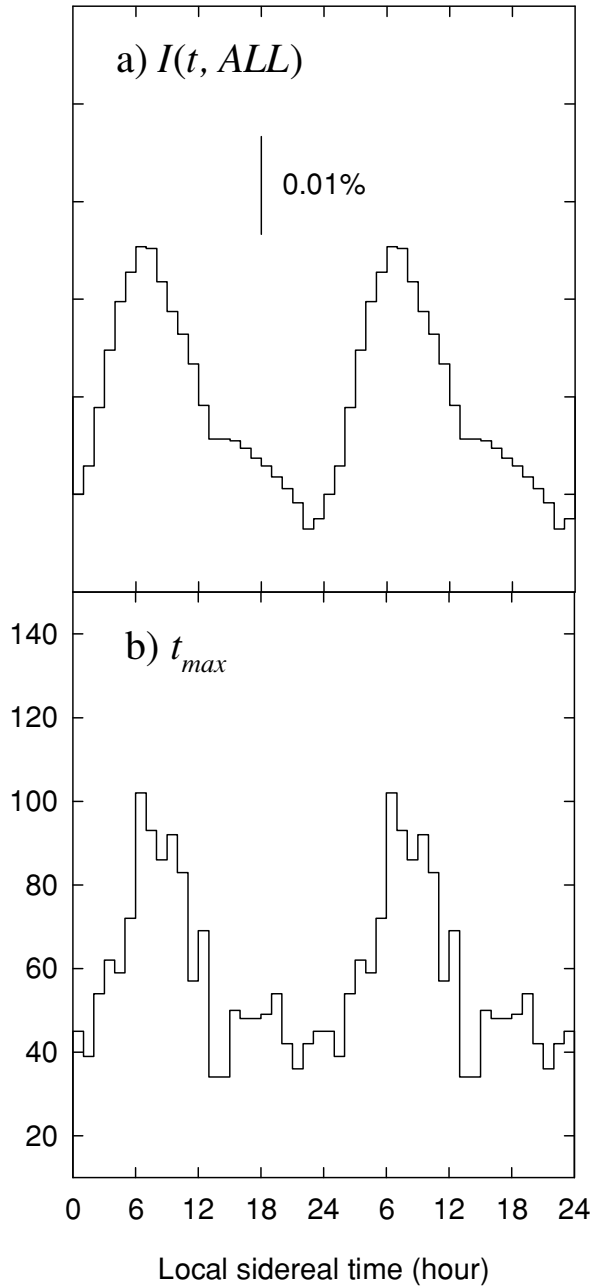

Fig. 1. a) Sidereal daily variation $I(t, A L L)$ of neutron intensity-The average of yearly variations $i(t)$ s observed at 74 stations in the period of 1960-2000. b) Histogram of maximum phase $t_{\max }$ of the first harmonic component of $i(t)$. The 24-hour time scale is repeatedly plotted so as to extend for a 2-day period.

narrowly recognized by the unnatural time profile in the time interval of $15<t \leq 21$ hours (cf. Fig. 1(a)). Originally, the appearance of this strange time profile gave us a clue to the discovery of a new anisotropy called the nose-in anisotropy from the direction of $\alpha \sim 18$ hours. The sidereal variation produced by this anisotropy is expressed as $H(t)$, whose time profile is shown in Fig. 2. The histogram of $t_{\max }$ in Fig. 1(b) also shows a characteristic hump in the time interval of $15<t \leq 21$ hours which supports the coexistence of $H(t)$ with $T(t)$.

The two anisotropies are variable in time and $H(t)$ occasionally becomes larger than or comparable with $T(t)$. Figure 2(a) shows respectively the apparently isolated $T(t)$ and $H(t)$, whereas they usually interact with each other. Figure 2(b) shows three kinds of interacting variations $i(t) \mathrm{s}$ with two peaks at $\sim 6$ and $\sim 18$ hours. These two peaks indicate that the variation is produced by two sharply concentrated fluxes from opposite directions. The example from Tokyo in 1986 in Fig. 2(b) contains also a first harmonic component with its maximum phase different from 6 and 18 hours, indicating that directions ( 6 and 18 hours) of two anisotropies are variable to some extent. The three exam- 


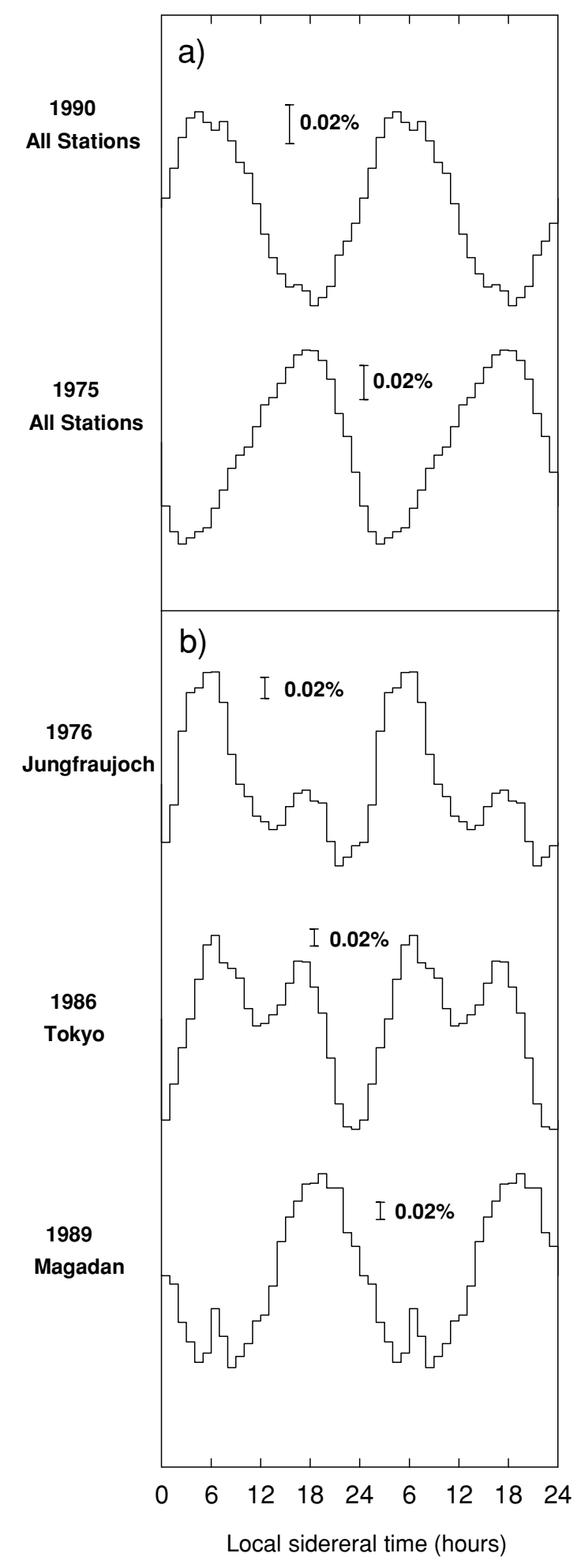

Fig. 2. a) Apprarently isolated $T(t, A L L)$ in 1990 and $H(t, A L L)$ in 1975. b) $i(t)$ s showing the coexistence of $T(t)$ and $H(t)$.

ples with the two peaks in Fig. 2(b) are presented to emphasize the coexistence of the anisotropies. Features like this, however, are not frequently observed and almost all the resultant variations $I(t) \mathrm{s}$ and $i(t) \mathrm{s}$ show one peak at $\sim 6$ or $\sim 18$ hours with a small mound or a gentle downward slope on either side of the peak like that seen in Fig. 1(a) or only a single peak with maximum phase different from 6 and 18 hours that results when two variations substantially overlap by the directional change of anisotropies. In order to obtain the origin of these anisotropies, the solar modulation of $T(t)$ and $H(t)$ is examined in the following sections.

\section{Solar Modulation of $I(t), T(t)$ and $H(t)$}

$I(t, A L L)$ in Fig. 1(a) is classified into $I(t, Y) \mathrm{s}$ in the four periods $(Y=A P, Q P, A N, Q N)$ as shown on the left-side of Fig. 3. $I(t, Y)$ s are mutually different in shape owing to the relative contribution between $T(t)$ and $H(t) . T(t)$ can be easily recognized in all the periods, especially in $A P$ period, but $H(t)$ is narrowly discernible only in $Q P$ and $Q N$ periods. In the following, an attempt is made to decompose $I(t, Y)$ into $T(t, Y)$ and $H(t, Y)$ on the assumption that $H(t, Y)$ is the resultant of yearly variations $i(t) \mathrm{s}$ whose first harmonic components have respectively their maximum phase in the time interval of $15<t \leq 21$ hours, during which the unfamiliar profiles of $I(t)$ and $t_{\max }$ in Fig. 1 are observed. The decomposed $H(t, Y)$ and $T(t$, $Y)$ are shown on the right-side of $I(t, Y)$. All the maximum phases of $T(t, Y)$ s show a remarkable coincidence with each other in contrast with the mutually scattered phases of $I(t, Y) \mathrm{s}$. The similarity between $T(t, Y \neq A P)$ and $T(t, A P)$ or $H(t, Y \neq A P)$ and $H(t, A P)$ is shown by the correlation diagram of their hourly values shown in Fig. 4. Their correlation coefficients are shown in Table 1 together with the first harmonic component of $T(t, Y)$ and $H(t, Y)$. These facts indicate that the above assumption for the decomposition into $T(t, Y)$ and $H(t, Y)$ would be justifiable. The characteristics of $T(t, Y)$ and $H(t, Y)$ are as follows.

1) $H(t, Y)$ is a little smaller than $T(t, Y)$ and they are about two times as large as $I(t, Y)$.

2) The occurrence rate $f_{H}(Y)$, which is the percentage of the number of $H(t, Y)$ in $Y$ period, is considerably smaller than $f_{T}(Y)$.

3) $f_{T}(Y)$ and $f_{H}(Y)$ are dependent on polarity state and solar activity.

4) $T(t, Y)$ and $H(t, Y)$ are almost independent of polarity state and solar activity.

The polarity independence of $T(t, Y)$ was found by the underground observation at Sakashita in 2004 and interpreted as being due to the acceleration of cosmic rays on the boundary of HMS (Nagashima et al., 2004). In the present analysis, this independence is observed not only in $T(t, Y)$ but also in $H(t, Y)$. Nevertheless, their resultant variation $I(t, Y)$ is subject to solar modulation. This is due to the variation of $f_{H}(Y)$ in different $Y$-period (cf. Table 1). In the quiet $(Q)$ period, $f_{H}(Q N)$ and $f_{H}(Q P)$ are approximately equal to each other, whereas, in the active (A) period, $f_{H}(A N)$ is larger than $f_{H}(A P)$. This indicates that $A P$ period activates $T(t)$ and/or inactivates $H(t)$; conversely $A N$ period activates $H(t)$ and/or inactivates $T(t)$, suggesting the polarity dependence of acceleration mechanisms producing these variations.

In the previous analysis of $T(t)$ observed with underground muon telescopes, the declination $\left(\delta_{R}\right)$ of the flux was estimated at $\sim-24^{\circ}$ from north-south asymmetry of 


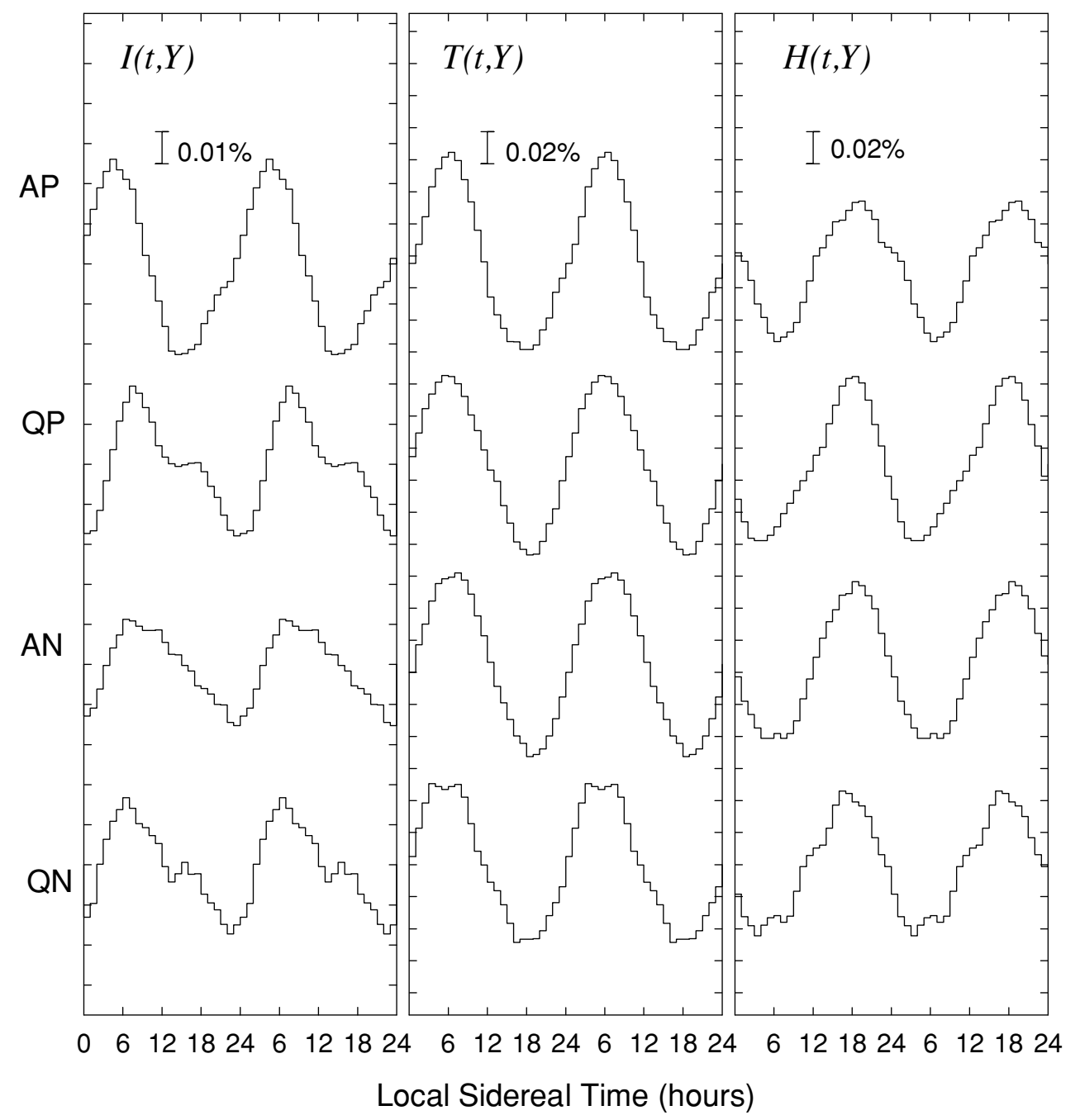

Fig. 3. $I(t, Y), T(t, Y)$ and $H(t, Y)$ in $A P, Q P, A N$ and $Q N$ period.

geographic latitude distribution of $T(t)$, (Ref. 1). In the present analysis, an attempt has been made to determine $\delta_{R}$ by using $i(t)$ s mainly produced by the tail-in or nose-in flux, but failed to do it owing to the non-uniform latitude distribution of stations and also to probably poor separation between $T(t)$ and $H(t)$. In the following, $\delta_{R}$ is assumed to be $0^{\circ}$ for both fluxes.

\section{Interpretation of Solar Modulation of $T(t)$ and $H(t)$}

5.1 Simulation of solar modulation of galactic anisotropy

Cosmic rays suffer magnetic deflection during their passage through HMS. This is called the solar modulation of cosmic rays. The modulation depends on the polarity state of the solar magnetic field at the north pole which changes its sign from positive to negative or vice versa every solar cycle and therefore produces a polarity-dependent sidereal daily variation if any cosmic-ray flux arrives at the Earth from a definite direction (hereafter called the principal direction of the flux). If the source flux $S(E, \chi)$ has a spatial distribution $F(\chi)$ expressed by the first order spherical function $P_{1}^{0}(\cos \chi)$ with the pitch angle $\chi$ measured from the principal direction $\left(\alpha_{R}, \delta_{R}\right)$, it is expressed by the series of spherical surface harmonics in the geographic polar coordinates $(r, \alpha, \pi / 2-\delta)$ as follows (cf. Nagashima et al., 1981, 1982),

$$
\begin{aligned}
S(E, \chi)= & \eta(E) F(\chi)=\eta(E) P_{1}^{0}(\cos \chi) \\
= & \eta(E) \sum_{m=0}^{1} P_{1}^{m}\left(\sin \delta_{R}\right) P_{1}^{m}(\sin \delta) \\
& \times \cos m\left(\alpha-\alpha_{R}\right),
\end{aligned}
$$

where $\eta(E)$ is the differential energy spectrum given by

$$
\eta(E)=\eta_{0}\left(E / 10^{2} \mathrm{GeV}\right)^{\gamma} .
$$

Hereafter, $S(E, \chi)$ is called the plane source model. If the principal direction is specified by $\alpha_{R}=6$ hours and $\delta_{R}=0^{\circ}, F(\chi)$ is reduced to

$$
F(\chi)=\cos \delta \sin \alpha .
$$

Owing to the cosmic-ray deflection in HMS, $F(\chi)$ produces at the boundary of the geomagnetosphere the following energy-dependent sidereal diurnal variation $D(t)$ observable with a detector with viewing direction $\delta_{0}$ in the meridian plane at a station.

$$
D(t)=a \cos 2 \pi\left(t-t_{\max }\right) / 24 \text { hours, }
$$


Table 1. Characteristics of $I(t, Y), T(t, Y)$ and $H(t, Y)$ for $Y=A P, Q P, A N$ and $Q N$ period.

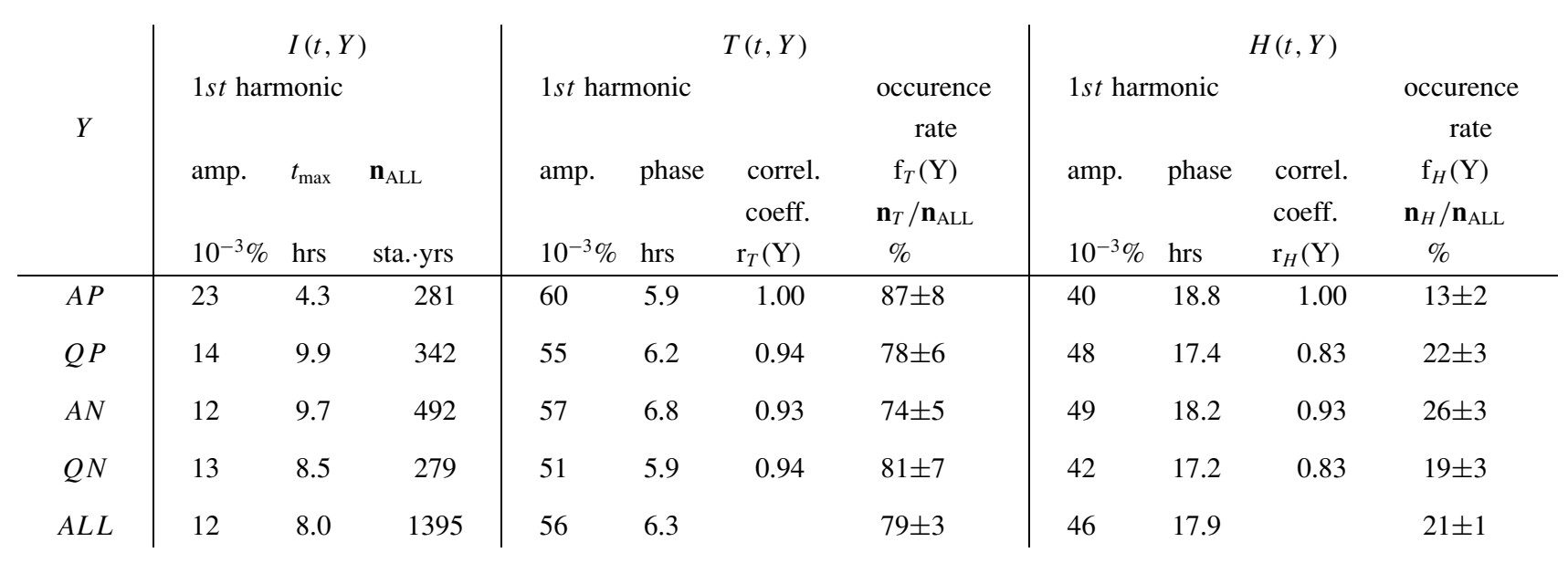
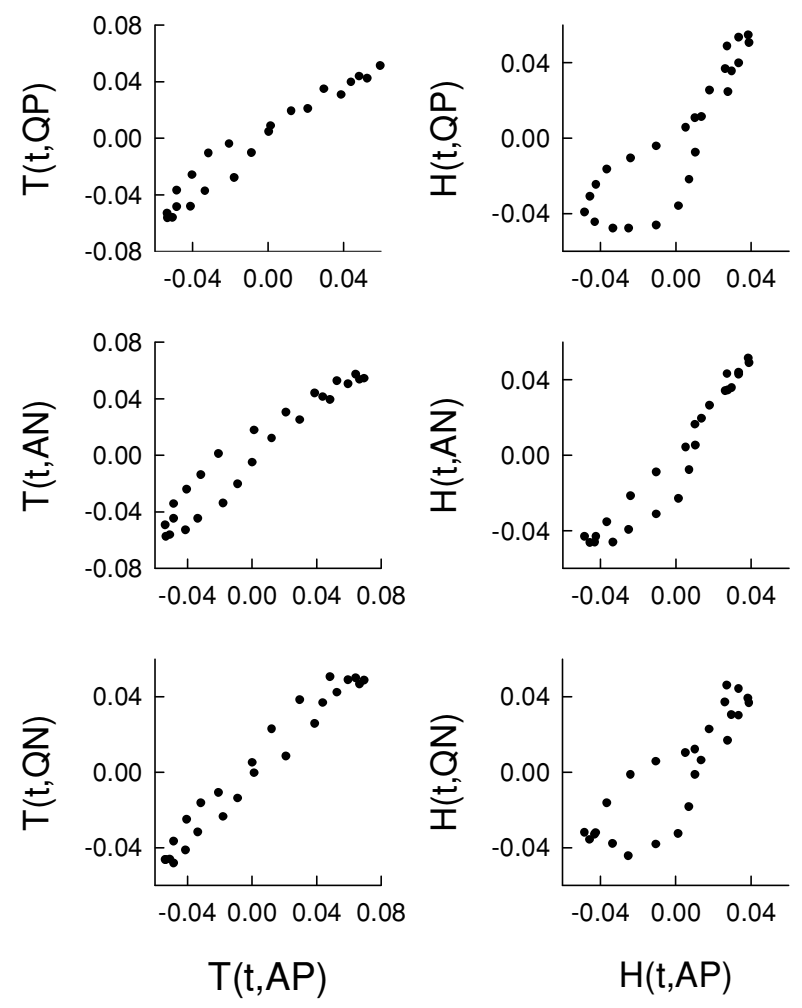

Fig. 4. Correlation diagram between $T(t, Y \neq A P)$ and $T(t, A P)$, and between $H(t, Y \neq A P)$ and $H(t, A P)$.

in which $a$ and $t_{\max }$ are functions of the following parameters; the cosmic-ray energy $(E)$, the polarity state (positive or negative), the interplanetary magnetic field $\left(B_{e}\right)$ at the heliocentric distance $r=1$ a.u., the heliolatitudinal amplitude $\left(\lambda_{W}\right)$ of the wavy neutral sheet and the scattering mean free path $l(r, E)$ of cosmic rays with $E$ in the irregular magnetic field at $r$ (cf. Yasue et al., 1983). Simulation of the solar modulation was made in the spherically symmetric HMS with the radius $10^{2}$ a.u. (Nagashima et al., 1981, 1982; Nagashima and Morishita, 1983). Following is an example of the simulation of the modulation of galactic anisotropy for $\delta_{0}=45^{\circ}$ in HMS with $B_{e}=4 \mathrm{nT}, \lambda_{W}=0^{\circ}$ and $l(r$,
$E)=\infty$. Figure 5 shows the energy dependence of $a$ and $t_{\max }$ of $D(t)$ in Eq/ (4). The simulation is applicable also to the case of $\alpha_{R}=18$ hours and $\delta_{R}=0^{\circ}$ by changing the phase from $t_{\max }$ to $t_{\max }+12$ hours. It is noted that $\left|\delta_{0}\right|=45^{\circ}$ in the above is nearly equal to the averaged geographic latitude $\left(|\lambda|=49^{\circ}\right)$ for all the neutron stations.

The polarity dependence of the variation in Fig. 5 can be interpreted as follows. Owing to the different motion of cosmic rays in the positive and negative polarity state in HMS with nearly concentric circular form in the region far away from the Sun, cosmic rays can arrive at the Earth mainly from the polar region $\left(|\delta| \gg 0^{\circ}\right)$ of HMS in the positive state and from the equatorial region $\left(\delta \sim 0^{\circ}\right)$ in the negative state (e.g. Nagashima et al., 1981). In the present case, as the principal direction is $\alpha_{R}=6$ hours and $\delta_{R}=0^{\circ}$, the Earth receives the flux with $\chi \sim 0^{\circ}$ from the equatorial direction $\left(\delta \sim 0^{\circ}\right)$ in the negative state whereas in the positive state, it receives the flux with $\chi \gg 0^{\circ}$ from the polar direction $\left(|\delta| \gg 0^{\circ}\right)$. Owing to the difference between $S\left(E, \chi \sim 0^{\circ}\right)$ and $S\left(E, \chi \gg 0^{\circ}\right)$, the sidereal variation is greater in the negative state than in the positive state.

The sidereal variation $\overline{D(t)}$ observable with the detector with viewing direction of $\delta_{0}=45^{\circ}$ on the Earth can be derived from a and $t_{\max }$ in Fig. 5 in considering the influence of the heliomagnetic statistical scatterings (cf. Yasue et al., 1985), the geomagnetic deflection and the atmospheric attenuation of cosmic rays. Table 2 shows the amplitude $\bar{a}$ and $\bar{t}_{\max }$ of $\overline{D(t)}$ produced by $S(E, \chi)$ with $\eta_{0}=1$ for $\gamma=0$ and -1 . The variations observable at Nagoya, Hobart and Sakashita stations also are derived from the same data in Fig. 5. As an index of the polarity dependence of the modulation, the following factor $R(N \mid P)$ is defined.

$$
R(N \mid P)=\overline{a(N)} / \overline{a(P)},
$$

where $\overline{a(N)}$ and $\overline{a(P)}$ are the amplitude of $\overline{D(t)}$ in the negative $(N)$ and positive $(P)$ polarity states. As the simulated $R(N \mid P)$ is larger than the observation's in low energies, $H(t)$ and $T(t)$ can not be produced by any excess flux of galactic origin. The only possible origin would be on the boundary of HMS where the interaction between the galactic and solar magnetic fields is thus believed to produce the 
Table 2. Amplitude $\bar{a}$ and phase $\bar{t}_{\max }$ of the simulated sidereal variation observable by a detector with viewing direction $\left|\delta_{0}\right|=45^{\circ}$ in the meridian plane at a station, produced by $S(E, \chi)$ with $\eta_{0}=1.0$ under the condition that $\alpha_{R}=6$ hours, $\delta_{R}=0^{\circ}, B_{e}=4 \mathrm{nT}, \lambda=0^{\circ}, l(r, E)=\infty$ and $\gamma=0$ and -1.0 .

\begin{tabular}{|c|c|c|c|c|c|c|c|c|c|}
\hline & & \multicolumn{2}{|c|}{ Neutron } & \multicolumn{2}{|c|}{ Nagoya } & \multicolumn{2}{|c|}{ Hobart } & \multicolumn{2}{|c|}{ Sakashita } \\
\hline & $E$ & \multicolumn{2}{|c|}{$20 \mathrm{GeV}$} & \multicolumn{2}{|c|}{$60 \mathrm{GeV}$} & \multicolumn{2}{|c|}{$184 \mathrm{GeV}$} & \multicolumn{2}{|c|}{$387 \mathrm{GeV}$} \\
\hline & $\lambda$ & \multicolumn{2}{|c|}{$49^{\circ}$} & \multicolumn{2}{|c|}{$35^{\circ}$} & \multicolumn{2}{|c|}{$43^{\circ}$} & \multicolumn{2}{|c|}{$36^{\circ}$} \\
\hline & \multirow[t]{2}{*}{$Y$} & $\bar{a}$ & $\bar{t}_{\max }$ & $\bar{a}$ & $\bar{t}_{\max }$ & $\bar{a}$ & $\bar{t}_{\max }$ & $\bar{a}$ & $\bar{t}_{\max }$ \\
\hline & & $10^{-2}$ & $\mathrm{hr}$ & $10^{-2}$ & $\mathrm{hr}$ & $10^{-2}$ & $\mathrm{hr}$ & $10^{-2}$ & $\mathrm{hr}$ \\
\hline \multirow[t]{2}{*}{$\gamma_{0}=0$} & $N$ & 6.6 & 5.8 & 12.7 & 5.6 & 30.1 & 6.0 & 43.0 & 5.8 \\
\hline & $P$ & 2.6 & 6.0 & 4.3 & 6.0 & 11.4 & 6.0 & 17.0 & 5.7 \\
\hline \multirow[t]{2}{*}{$\gamma_{0}=-1$} & $N$ & 2.4 & 5.3 & 5.8 & 5.0 & 10.9 & 5.5 & 12.0 & 5.4 \\
\hline & $P$ & 0.55 & 7.1 & 2.0 & 6.2 & 2.4 & 6.5 & 3.0 & 6.2 \\
\hline
\end{tabular}
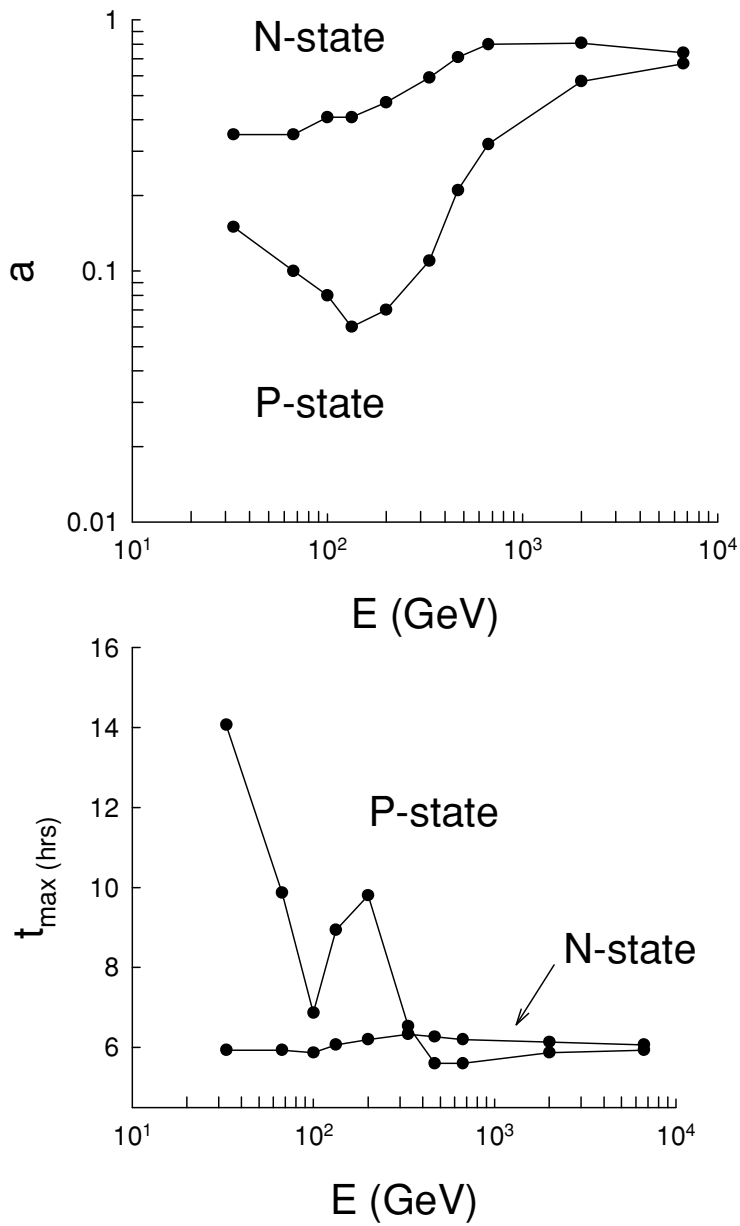

Fig. 5. Energy dependence of amplitude $a$ and maximum phase $t_{\max }$ of the simulated sidereal diurnal variation observable on the geomagnetospheric boundary by a detector with viewing direction $\left|\delta_{0}\right|=45^{\circ}$ in the meridian plane at a station, produced by $S(E, \chi)$ in Eq. (1) under the condition that $\eta_{0}=1.0, \alpha_{R}=6$ hours, $\delta_{R}=0^{\circ}, B_{e}=4 \mathrm{nT}, \lambda_{W}=0^{\circ}$ and $l(r, E)=\infty$.

cosmic-ray acceleration.

\subsection{Source flux on the boundary of HMS}

If the source flux is localized in a small surface region of the nose head and tail end boundary of HMS, $H(t)$ and $T(t)$ can not be observed at the Earth in the positive polarity state, because, as pointed out previously, almost all the cosmic rays except for those insensitive to the magnetic field can not arrive at the Earth from the above source region in the positive state. Therefore, for the explanation of the polarity-independent variation, the source must be distributed on all the boundary surface at the least. This is called hereafter the curved-surface source model in contrast with the previous plane source model.

\section{a) Source for $H(t)$}

The source flux, which is distributed on the hemispherical surface of the helionose boundary with its centre at $\alpha=18$ hours and $\delta=0^{\circ}$, is expressed as $S_{H}\left(E, \chi, \boldsymbol{n}_{R}\right.$, $\left.\alpha_{b}, \delta_{b}\right)$ in which the unit vector $\boldsymbol{n}_{R}$ expresses the principal direction of the flux at a point $\left(\alpha_{b}, \delta_{b}\right)$ on the hemisphere. In the following, the magnitude $\eta_{H}(E)$ of $S_{H}\left(E, \chi, \boldsymbol{n}_{R}, \alpha_{b}\right.$, $\delta_{b}$ ) is assumed for the moment to be constant on all the surface and equal to $\eta_{G}(E)$ of $S_{G}(E, \chi)$ of galactic origin in Eq. (1). As the acceleration mechanism is not known, the direction of $\boldsymbol{n}_{R}$ is not certain but probably antiparallel to the motion $(\boldsymbol{v})$ of the solar system or parallel to the normal $\left(\boldsymbol{n}_{b}\right)$ of the boundary surface. In the case of $\boldsymbol{n}_{R} / /-\boldsymbol{v}, S_{H}(E, \chi$, $\left.\boldsymbol{n}_{R}, \alpha_{b}, \delta_{b}\right)$ coincides with $S_{G}(E, \chi)$ as above and therefore the simulation using the plane source model is applicable here. In the case of $\boldsymbol{n}_{R} / / \boldsymbol{n}_{b}$, on the other hand, although the overall similarity between the two sources does not exist, their partial similarity can be found near the equatorial region $\left(\delta \sim 0^{\circ}\right)$. Therefore, in the negative $(N)$ state in both cases of $\boldsymbol{n}_{R}$, the magnitude $\eta_{H}(E)$ of $S_{H}\left(E, \chi, \boldsymbol{n}_{R}, \alpha_{b}, \delta_{b}\right)$ near the equatorial region can be exactly or approximately obtained from $H(t, N) \sim 0.1 \%$ with the aid of the simulation, as

$$
\eta_{H}\left(10^{2} \mathrm{GeV}\right)= \begin{cases}1.5 \% & \text { for } \gamma=0 \\ 4.2 \% & \text { for } \gamma=-1 .\end{cases}
$$

The energy gain $(\Delta E)$ of cosmic rays by the acceleration required for producing $\eta_{H}\left(10^{2} \mathrm{GeV}\right)$ is estimated by the following equation.

$$
\eta_{H}(E)=(2+|\Gamma|) \cdot(\Delta E / E)
$$

where $\Gamma(-2.6)$ is the power exponent of the differential energy spectrum of galactic cosmic rays. As the acceleration on the boundary would be independent of $E, \eta_{H}$ is proportional to $E^{-1}$. In this respect, the magnitude for $\gamma=-1$ in Eq. (6) is preferable and it requires the following energy gain.

$$
\Delta E=0.9 \mathrm{GeV} \text {. }
$$


It is noted that this value is however only a rough estimate as it would be considerably changed by the alteration of $l(r$, $E)$ and $\lambda_{W}$.

In the positive $(P)$ state, on the other hand, the flux arrives at the Earth from the high declination $\left(|\delta| \gg 0^{\circ}\right)$. Generally, the flux from the direction of high declination $\left(|\delta| \gg 0^{\circ}\right)$ produces less sidereal variation than that from the direction of $\delta \sim 0^{\circ}$. Therefore, whatever the modulation of the flux in the magnetosphere may be, the variation in the positive state would never become greater than or comparable with that in the negative state, if the source flux is uniformly distributed on the hemisphere. For the explanation of the polarity independence of $H(t)$, it is necessary to assume that the source flux increases with the increase of $|\delta|$.

\section{b) Source for $T(t)$}

The source flux $S_{T}\left(E, \chi, \boldsymbol{n}_{R}, \alpha_{b}, \delta_{b}\right)$ is distributed on the cylindrical surface of heliotail with its principal direction $\boldsymbol{n}_{R}$ parallel to $\boldsymbol{n}_{b}$. As before, the source flux arrives from the equatorial region $\left(\delta \sim 0^{\circ}\right)$ in the negative state and from the polar region $\left(|\delta| \gg 0^{\circ}\right)$ in the positive state, but the behavior of the flux on the cylindrical boundary is modified as follows. In the positive $(P)$ state, the principal direction $\boldsymbol{n}_{R}$ perpendicular to the cylindrical surface in the polar region $\left(|\delta| \gg 0^{\circ}\right)$ is deflected toward the Earth, as the vector product $\left[\boldsymbol{n}_{R} \times \boldsymbol{B}\right]$ between $\boldsymbol{n}_{R}$ and the magnetic field $(\boldsymbol{B})$ is directed toward the Earth. Therefore, almost all the flux $S_{T}\left(E, \chi, \boldsymbol{n}_{R}, \alpha_{b}, \delta_{b}\right)^{\text {po }}$ on the polar (po) boundary in the positive $(P)$ state can contribute to the variation. On the contrary, in the negative $(N)$ state, the principal direction of the flux $S_{T}\left(E, \chi, \boldsymbol{n}_{R}, \alpha_{b}, \delta_{b}\right)^{\text {eq }}$ from the equatorial (eq) region $\left(\delta \sim 0^{\circ}\right)$ can not be deflected toward the Earth as $\left[\boldsymbol{n}_{R} \times \boldsymbol{B}\right]$ is not toward the Earth. Therefore, only partial flux with large $\chi$ on the earth-side can contribute to the variation. In addition to these sources, the heliotail contains the tail-end sources $S_{T}\left(E, \chi, \boldsymbol{n}_{R}, \alpha_{b}, \delta_{b} \mid P\right)^{\text {en }}$ and $S_{T}(E$, $\left.\chi, \boldsymbol{n}_{R}, \alpha_{b}, \delta_{b} \mid N\right)^{\text {en }}$ in the positive $(P)$ and negative $(N)$ states. Therefore, the heliotail produces the following two kinds of sources in the positive and negative states.

$$
\begin{aligned}
& \qquad \begin{aligned}
S_{T}(E, \chi & \left.\boldsymbol{n}_{R}, \alpha_{b}, \delta_{b} \mid P\right)=S_{T}\left(E, \chi, \boldsymbol{n}_{R}, \alpha_{b}, \delta_{b} \mid P\right)^{\mathrm{po}} \\
& +S_{T}\left(E, \chi, \boldsymbol{n}_{R}, \alpha_{b}, \delta_{b} \mid P\right)^{\mathrm{en}},
\end{aligned} \\
& \text { and } \\
& \qquad \begin{aligned}
S_{T}(E, \chi, & \left.\boldsymbol{n}_{R}, \alpha_{b}, \delta_{b} \mid N\right)=S_{T}\left(E, \chi, \boldsymbol{n}_{R}, \alpha_{b}, \delta_{b} \mid N\right)^{\mathrm{eq}} \\
& +S_{T}\left(E, \chi, \boldsymbol{n}_{R}, \alpha_{b}, \delta_{b} \mid N\right)^{\mathrm{en}} .
\end{aligned}
\end{aligned}
$$

and

The contribution of these fluxes to the variation depends on the length $(L)$ of the cylinder. With the decrease of $L$, the cylindrical heliotail tends to the hemisphere. On the other hand, with the increase of $L$, the contribution of $S_{T}(E, \chi$, $\left.\boldsymbol{n}_{R}, \alpha_{b}, \delta_{b} \mid P\right)^{\mathrm{en}}$ and $S_{T}\left(E, \chi, \boldsymbol{n}_{R}, \alpha_{b}, \delta_{b} \mid N\right)^{\mathrm{eq}}$ are reduced in turn owing to the reduction of the solid angle of tail-end surface seen from the Earth, and consequently the contribution of the fluxes on the cylindrical surface becomes predominant. Although the modulation of these fluxes is not known, the polarity independence of the variation could be more easily realized than that of the nose-in flux, because the contribution of $S_{T}\left(E, \chi, \boldsymbol{n}_{R}, \alpha_{b}, \delta_{b} \mid P\right)^{\text {po }}$ to the variation is greater than that of $S_{T}\left(E, \chi, \boldsymbol{n}_{R}, \alpha_{b}, \delta_{b} \mid N\right)^{\mathrm{eq}}$ even if the two source fluxes are equal to each other. This difference of the acceleration mechanisms on the tail and nose boundary would be one of the causes producing the superiority of $T(t)$ in magnitude over $H(t)$ in Fig. 1(a).

\section{Discussion}

$H(t)$ and $T(t)$ coexist almost all the time. Their separation has been made by using the characteristic time distribution of $I(t)$ and $t_{\max }$ in Fig. 1. The separated $H(t)$ and $T(t)$, however, do not express their absolute magnitudes as can be seen by the following examples. According to the criterion for the separation, the variations $i(t) \mathrm{s}$ at Jungfraujoch and Tokyo in Fig. 2(b) are classified into $T(t)$. As these variations contain also the non-negligible variation of the nose origin, the magnitude of $T(t)$ is reduced by this contamination. The contamination would be proportional to the occurrence rate of the other variation in Table 1 and therefore would be greater in $H(t)$ than in $T(t)$. This would cause less correlation coefficient $r_{H}(Y)$, (cf. Table 1). The absolute magnitude of $T(t)$ and $H(t)$ cannot be obtained unless the detector approaches their source regions as close as possible.

In the simulation of the solar modulation of cosmic-ray anisotropy, the boundary of HMS was assumed to be spherical, and its magnetic structure was not taken into consideration. Although the structure might be closely related to the acceleration of cosmic rays, it would not affect the propagation of cosmic rays through HMS. The simulation depends on the parameters of HMS such as the magnetic strength, the cosmic-ray scattering mean free path $l(r, E)$ and so forth as described previously. Among these parameters, $l(r, E)$ is the most uncertain parameter in high energy region $(E \gg 15 \sim 20 \mathrm{GeV})$. The formula of $l(r$, $E$ ) used in the present simulation was assumed by Yasue $e t$ al. (1983) on the basis of the information on $l(r=1$ a.u.; $E \leq 15 \sim 20 \mathrm{GeV})$. The amplitude of the variation is sensitive to the change of $l(r, E)$ in low energy region $(<\sim 300$ $\mathrm{GeV})$, but $R(N \mid P)$ does not change so much as the polarity dependence of the scattering effect is not so large (cf. Yasue et al., 1983). The amplitude is affected also by the wavy neutral sheet, and decreases with the increase of $\lambda_{W}$, but $R(N \mid P)$ is always greater than unity (cf. Nagashima and Morishita, 1983). As above, the alteration of the parameters does not require any change of the previous conclusion as to the origin of $H(t)$ and $T(t)$ that they are produced by the acceleration of cosmic rays on the boundary surface of HMS.

It is noted here that as far as we assume such a surface acceleration, there would be no reason to deny the acceleration on the surface in the intermediate region between the nose and tail boundaries, which would produce sidereal variations with the maximum phase at $\sim 0$ hours and $\sim 12$ hours, respectively. If these two fluxes $(\sim 0$ hours, $\sim 12$ hours) would equally exist with those from the nose and tail directions ( $\sim 6$ hours and $\sim 18$ hours), there would be no sidereal variation observable at the Earth. The appearances of $H(t)$ and $T(t)$ produced by the only latter two fluxes would be due to the geometrical asymmetry of HMS with the nose and tail boundaries where two different types of acceleration would occur as has been illustrated in the previous section. The effect of the different accel- 
eration mechanism can be inferred from the difference between the occurrence ratios $f_{H}(Y)$ and $f_{T}(Y)$ in Table 1 and also from the enhancement of $T(t)$ in $I(t)$ in Fig. 1(a). It is noted also that the present analysis has not been able to detect the sidereal variation $G(t)$ of galactic origin. $G(t)$ observed in higher energy region has a plateau-like shape with a sharp and deep sink at $\sim 12$ hours (cf. Nagashima et al., 1998, 2004; Ref. 1). On the contrary, $I(t)$ in Fig. 1 does not contain such a variation as it has the minimum intensity at $\sim 22.5$ hours which almost coincides with the center $(\sim 0$ hours) of the plateau of $G(t)$. This seems to suggest that $G(t)$ does not exist at the low energies owing to severe solar modulation or would be submerged beneath $I(t)$ even if it were to exist.

The polarity independence of $H(t)$ and $T(t)$ expressed by $R(N \mid P) \sim 1$ can be realized by compensating the influence of the polarity-dependent solar modulation with the declination-dependent source flux. As the accelerations, producing the source fluxes in the nose and tail boundaries, are different from each other, the solar modulations in the nose and tail side HMS must be different from each other to produce respectively the polarity independences of $T(t)$ and $H(t)$. The polarity independence is also observed in $T(t)^{\mathrm{SAK}}$ in the energy region of $\sim 400 \mathrm{GeV}$ at Sakashita underground station and shows almost the same value as that of $T(t)$, as (cf. Nagashima et al., 2004).

$$
R_{T}(N \mid P)^{\mathrm{SAK}} \sim 1.1 \quad \text { at } E_{m} \sim 400 \mathrm{GeV} .
$$

In order to explain the same ratio $R(N \mid P)$ in low and high energy regions, the polarity-dependence of solar modulation must be proportional to $E^{-1}$ as the source flux produced by the acceleration is supposed to be proportional to $E^{-1}$.

It would be reasonable to presume that the acceleration depends on solar activity. Contrary to this presumption, the observed $H(t)$ and $T(t)$ do not show such an activity dependence (cf. Table 1). On the other hand, according to the muon observation at Sakashita underground station in the energy region of $\sim 400 \mathrm{GeV}, T(t)^{\mathrm{SAK}}$ shows the following activity dependence, definitely supporting the above presumption (Nagashima et al., 2004).

$$
\begin{aligned}
& T(t, A P)^{\mathrm{SAK}} \sim 1.5 \cdot T(t, Q P)^{\mathrm{SAK}}, \\
& T(t, A N)^{\mathrm{SAK}} \sim 1.8 \cdot T(t, Q N)^{\mathrm{SAK}} .
\end{aligned}
$$

The reason for no response of neutron intensity to the activity is not certain but might be due to the error caused by the interaction between $T(t)$ and $H(t)$.

Finally, the observations in high and low energies show the following unconventional energy dependence of $T(t)$. The magnitude of $T(t, A P)$ in Fig. 3 measured from the top to the bottom is $\sim 0.1 \%$ and larger than that $(0.08 \%)$ of $T(t, A P)^{\mathrm{SAK}}$ observed with $S$-component of muon telescope $\left(\lambda=5^{\circ} \mathrm{S}, E_{m}=387 \mathrm{GeV}\right)$ at Sakashita station (cf. Nagashima et al., 2004). This suggests that the energy spectrum of the tail-in anisotropy responsible for $T(t)$ would be of power-exponent type with a negative exponent. Such an energy spectrum with a negative exponent was not observed in the energy region less than $\sim 10^{4} \mathrm{GeV}$ before 1998 when the observed sidereal variations were thought to be produced by a single galactic anisotropy and even after 1998 when these variations were found to be decomposed into two kinds of variation $G(t)$ and $T(t)$, (cf. Ref. 1). The negative spectrum of $T(t)$ has been found by the present analysis owing to the separation between $T(t)$ and $H(t)$ in the variation observed by the neutron monitors. This spectrum seems to support the hyposesis of cosmic-ray acceleration on the heliotail boundary for the explanation of the solar modulation of the sidereal variation observed at Sakashita in the previous paper (Nagashima et al., 2004), as the acceleration is supposed to be more effective in lower energy region. However, it is not certain whether such an excess flux in high energy region $(\sim 20 \mathrm{GeV} \sim 400 \mathrm{GeV})$ could be related to the accelerated galactic cosmic rays in low energy region $(\ll \sim 1 \mathrm{GeV})$ observed near the heliospheric termination shock by Voyager I (McDonald et al., 2003) and also whether it could be theorertically explained by the solar modulation of galactic cosmic rays based on the Parker's (1963) cosmic-ray transport equation, so far derived by several authors (e.g. Jokipii et al., 1993; Caballero-Lopez et al., 2004). The gradient of the radial density distribution of cosmic rays in HMS obtained by these authors produces the excess flux (the current) of cosmic rays, which is obserbed as a solar daily variation with the revolution of the Earth on its axis. If the density gradient $(\Delta)$ is symmetric with respect to the right ascension $\alpha$, the solar daily variaton is constant through one year. But, if $\Delta$ depends on $\alpha$, the variation is subject to seasonal variation, which can be observed as a sidereal daily variation. For the explanation of the predominance of $T(t)$ over $H(t)$, it is necessary to assume, for example, the following $\Delta(\alpha)$.

$$
\Delta(\alpha)=\delta_{0}+\delta(\alpha) \cos ^{2}\{2 \pi(\alpha-6 h r) / 24 \mathrm{hr}\},
$$

where

$$
\delta(\alpha)=\delta_{1}[1+\varepsilon \cos \{2 \pi(\alpha-6 h r) / 24 \mathrm{hr}\}],
$$

in which

$$
\delta_{0}>0, \quad \delta_{1}>0 \quad \text { and } 1>\varepsilon>0 .
$$

The coefficients in Eq. (14) must be variable depending on the polarity state $(P$ or $N)$ and the solar activity ( $A$ or $Q$ ). If $\varepsilon=0, \Delta(\alpha)$ produces equal peaks at $\alpha=6$ hours and 18 hours.

The $\alpha$-dependent gradient can not be produced by each kind of any magnetic disturbances such as the magnetic shock wave and so forth, because the location and the occurrence time of their source on the solar surface are quite arbitary and do not show any $\alpha$-dependence. These disturbances can not produce the $\alpha$-dependence even if they interact with the interplanetary magnetic field within a region showing a rotational symmetry with respect to the solar rotation axis. The only possible origin producing the required $\alpha$-dependent gradient would be on the outermost boundary region of HMS with the nose and tail structure which is produced by the motion of the solar system relative to the interstellar magnetic field.

\section{Conclusion}

Coexistence of two kinds of sidereal daily variations $H(t)$ and $T(t)$ produced respectively by sharply concentrated excess fluxes of cosmic rays from the nose and tail 
directions (right ascension $\alpha \sim 18$ and $\sim 6$ hours) of HMS was found by the analysis of neutron intensities (median energy $E_{m} \sim 20 \mathrm{GeV}$ ) on the ground in the period of 1960 2000. These variations have the following characteristics.

1) Polarity-independence- $H(t)$ and $T(t)$ as well as $T(t)^{\mathrm{SAK}}$ are independent of the polarity reversal of the solar magnetic field at the north pole. This is contradictory to the simulation of the solar modulation of galactic anisotropy, which produces sidereal variation at the Earth greater in the negative polarity state than in the positive state. Such excess fluxes would be produced on the nose and tail boundaries of HMS where the interaction between the galactic and solar magnetic fields are thought to produce the cosmic-ray acceleration.

2) Solar activity dependence- $T(t)^{\mathrm{SAK}}$ becomes larger in the active $(A)$ period than in the quiet $(Q)$ period of solar cycle. This is reasonable as the source is produced by the acceleration of cosmic rays caused by the interaction between the galactic and solar magnetic fields on the boundary surface of HMS. On the contrary, $T(t)$ and $H(t)$ do not clearly show such a dependence but only a slight tendency. The reason for it is not certain but might be due to incomplete separation of $T(t)$ and $H(t)$.

3) Energy dependence- $T(t)$ is larger than $T(t)^{\mathrm{SAK}}$. This indicates that the source flux would have an energy spectrum $\left(E^{\gamma}\right)$ with negative exponent $(\gamma<0)$ and would be moderately modulated in HMS so that the sidereal variation at the Earth could still retain the energy spectrum with an exponent $\left(\gamma^{\prime}\right)$ less than zero. The acceleration of cosmic rays could produce such a spectrum, but as for the modulation it is not certain whether or not the condition would be satisfied.

4) Location of source activity-Although the acceleration of cosmic rays might occur all over the boundary surface, it can be observed only from the directions of $\sim 6$ hours and $\sim 18$ hours. This would be due to the difference of the acceleration activity caused by the geometrical asymmetry of HMS with nose and tail boundaries. The acceleration would be more active on the tail boundary than on the nose boundary and most active on the polar surface $\left(|\delta| \gg 0^{\circ}\right)$ of the cylindrical tail boundary so that $T(t, A L L)$ predominates in $I(t, A L L)$ in Fig. 1(a). In addition to these requirements, the source activity on the nose and tail boundaries must be stronger in the high declination region $\left(|\delta| \gg 0^{\circ}\right)$ than in the equatorial region in order to realize the polarity independence of $T(t)$ ad $H(t)$ against solar modulation.

Acknowledgments. The authors express their sincere appreciation to the researchers at neutron monitor stations who have continued to contribute their precious data to WDC for Cosmic Rays for long-term period and also to the staffs of the former WDC-C2 for Cosmic Rays at Institute of Physical and Chemical Research, Tokyo, and the present WDC-C2 at Solar-Terrestrial Environment Laboratory, Nagoya University/Department of Environmental Sci- ences, Ibaraki University for the compilation of the neutron monitor data used here. The authors thank also to the members of Cosmic Ray Section, Solar-Terrestrial Environment Laboratory, Nagoya University for the kind discussions.

\section{References}

Bercovitch, M., The response of the cosmic ray sidereal diurnal variation to reversal of the solar magnetic field, Proc. International Symposium on Cosmic Ray Modulation in the Heliosphere, 329-336, Iwate Univ., Morioka, Japan, 1984.

Caballero-Lopez, R. A., H. Moraal, and F. B. McDonald, Galactic cosmic ray modulation : effects of the solar wind termination shock and the heliosheath, J. Geophys. Res., 109, A05105, doi:10.1029/2003JA010358, 2004.

Fenton, A. G., R. M. Jacklyn, and R. B. Taylor, Cosmic ray observations at 42 mwe underground at Hobart, Tasmania, Il. Nuovo Cimento, 22, 3985-3996, 1961.

Howard, R., Studies of solar magnetic fields I: The average field strength. Solar Phys., 38, 283-299, 1974.

Jacklyn, R. M., Evidence for a two-way sidereal anisotropy in the charged primary cosmic radiation, Nature, 211, 690-693, 1966.

Jacklyn, R. M., Galactic cosmic ray anisotropies in the energy range $10^{11}$ $10^{14}$ eV., Proc. Astron. Soc. Australia, 6, 425-436, 1986.

Jokipii, J. R., J. Kota, and E. Merenyi, The gradient of galactic cosmic rays at the solar-wind termination shock, Astrophys. J., 405, 782-786, 1993.

Kiraly, P., J. Kota, J. L. Osborne, N. S. Stepley, and A. W. Wolfendale, The anisotropy of cosmic ray from $10^{11}$ to $10^{20} \mathrm{eV}$, Riv. Nuovo Cimento, 2(2), 1-46, 1979.

McDonald, F. B., E. C. Stone, A. C. Cummings, B. Heikkila, N. Lal, and W. R. Webber, Enhancements of energetic particles near the heliospheric termination shock, Nature, 426, 48-51, 2003.

Mori, S., S. Yasue, S. Sagisaka, M. Ichinose, K. Chino, S. Akahane, and T. Higuchi, Matsushiro underground cosmic-ray observation (220 m.w.e. depth) and the observation of high energy $\left(\# 10^{12} \mathrm{eV}\right)$ cosmic ray intensity variation, J. Fac. Sci., Shinshu Univ., Matsumoto, Japan., 24(1), 1989.

Nagashima, K. and S. Mori, Summary of general discussion on sidereal daily variation of high energy cosmic rays, Proceedings of International Cosmic Ray Symposium on High Energy Cosmic Ray Modulation, Tokyo Univ., Japan, 326-360, 1976.

Nagashima, K. and I. Morishita, Cosmic ray sidereal daily variation of galactic origin observable in the heliomagnetosphere, Rep. of CosmicRay Research Laboratory, Nagoya Univ., Nagoya, Japan, no. 8, 1983.

Nagashima, K., I. Morishita, and S. Yasue, Asymptotic orbits of cosmic rays incident on the Earth from galactic space, Proc. 17th International Cosmic Ray Conference, 4, 189-192, 1981.

Nagashima, K., I. Morishita, and S. Yasue, Modulation of galactic cosmic ray anisotropy in the heliomagnetosphere: Average sidereal daily variation, Planet. Space Sci., 30, 879-896, 1982.

Nagashima, K., Y. Ishida, S. Mori, and I. Morishita, Cosmic ray sidereal diurnal variation of galactic origin observed by neutron monitors, Planet. Space Sci., 31, 1269-1278, 1983.

Nagashima, K., K. Fujimoto, and R. M. Jacklyn, The excess influx of galactic cosmic rays from the tailend side of the heliomagnetosphere, inferred from their sidereal daily variation, Proceedings of International Mini-Conference on Solar Particle Physics and Cosmic Ray Modulation, Solar-Terrestrial Environment Laboratory, Nagoya Univ., Nagoya, Japan, 93-98, 1995.

Nagashima, K., K. Fujimoto, and R. M. Jacklyn, Galactic and heliotail-in anisotropies of cosmic rays as the origin of sidereal daily variation in the energy region < $10^{4} \mathrm{GeV}$, J. Geophys. Res., 103, 17429-17440, 1998.

Nagashima, K., Z. Fujii, and K. Munakata, Solar modulation of galactic and heliotail-in anisotropies of cosmic rays at Sakashita underground station $(320 \sim 650 \mathrm{GeV})$, Earth Planets Space, 56, 479-483, 2004.

Parker, E. N., Interplanetary Dynamic Process, pp. 115-128, Interscience Division, John Wiley and Sons, New York, 1963.

Yasue, S., I. Morishita, and K. Nagashima, Modulation of galactic cosmic ray anisotropy in heliomagnetosphere: influence of cosmic ray scattering on sidereal daily variation, Planet. Space Sci., 33, 1057-1068, 1983.

K. Nagashima, I. Kondo (e-mail: ikrabbit@jcom.home.ne.jp), and Z. Fujii (e-mail: fujii@stelab.nagoya-u.ac.jp) 